\title{
Nocardia neocaldoniensis as a Cause of Skin and Soft Tissue Infection
}

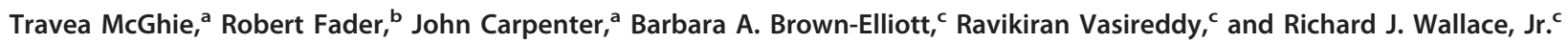 \\ Department of Medicine, Division of Infectious Disease, ${ }^{a}$ and Department of Pathology, Scott \& White Healthcare, ${ }^{\mathrm{b}}$ Texas A\&M School of Medicine, Temple, Texas, USA, \\ and Mycobacteria/Nocardia Laboratory, The University of Texas Health Science Center, Tyler, Texas, USA
}

\begin{abstract}
Nocardia neocaledoniensis was introduced as a new environmental species of Nocardia in 2004. We present the first case of human skin and soft tissue infection caused by this species in a patient with rheumatoid arthritis receiving prednisone and methotrexate therapy.
\end{abstract}

\section{CASE REPORT}

68-year-old man with a history significant for rheumatoid arthritis receiving prednisone and methotrexate therapy for at least 10 years presented for evaluation of a persistent right facial abscess. The patient lived in a house in a residential area in Central Texas, was a retired telephone employee, and volunteered as a firefighter, with moderate exposure to dirt. His hobbies included hunting, fishing, and mowing the lawn. He had no recent travel outside his hometown, and no history of opportunistic infections.

Approximately 1 month prior to presentation, he noticed right facial discomfort and abscess formation in his right jaw. His primary care physician prescribed cephalexin, but the patient had minimal relief of symptoms. The patient was referred to an otolaryngologist and a specimen was obtained by fine-needle aspiration. The Gram stain of the specimen revealed many polymorphonuclear leukocytes, but no organisms were observed. The corresponding culture recovered an organism suggestive of $\mathrm{No}$ cardia species. The patient was started on oral trimethoprim-sulfamethoxazole therapy, and his methotrexate treatment was discontinued. The patient underwent incision and drainage 9 days later due to increased drainage, and the resulting specimen once again revealed a Nocardia species with susceptibilities similar to those of the first isolate, as well as the presence of coagulase-negative staphylococci. The patient reported that he was compliant with trimethoprim-sulfamethoxazole; however, 22 days after the initial drainage, additional masses were noted in the same region. A magnetic resonance image (MRI) of the brain was negative for acute intracranial abnormalities.

On the basis of continued infection despite oral outpatient antibiotic therapy, the patient was admitted to the hospital. On admission, the patient denied cardiac, pulmonary, and gastrointestinal symptoms. On physical examination, his left submandibular gland was normal but the right submandibular triangle had a large mass (10.5 cm diameter) adjacent to the inferior border of the mandible. The area was erythematous and tender to palpation. Further examination of the right angle of the jaw submaxillary triangle area revealed two additional masses, one anterior and one posterior to the previous incision area. Both were firm and extremely tender to touch. Imaging of the neck and lungs did not reveal any sinus tract or acute pulmonary abnormalities. The patient was placed on intravenous imipenem and oral trimethoprim-sulfamethoxazole therapy. He underwent subsequent incision and drainage of the multiple skin abscesses. The patient's hospital course was complicated by acute renal insufficiency and hyponatremia, and his antibiotics were later switched to oral

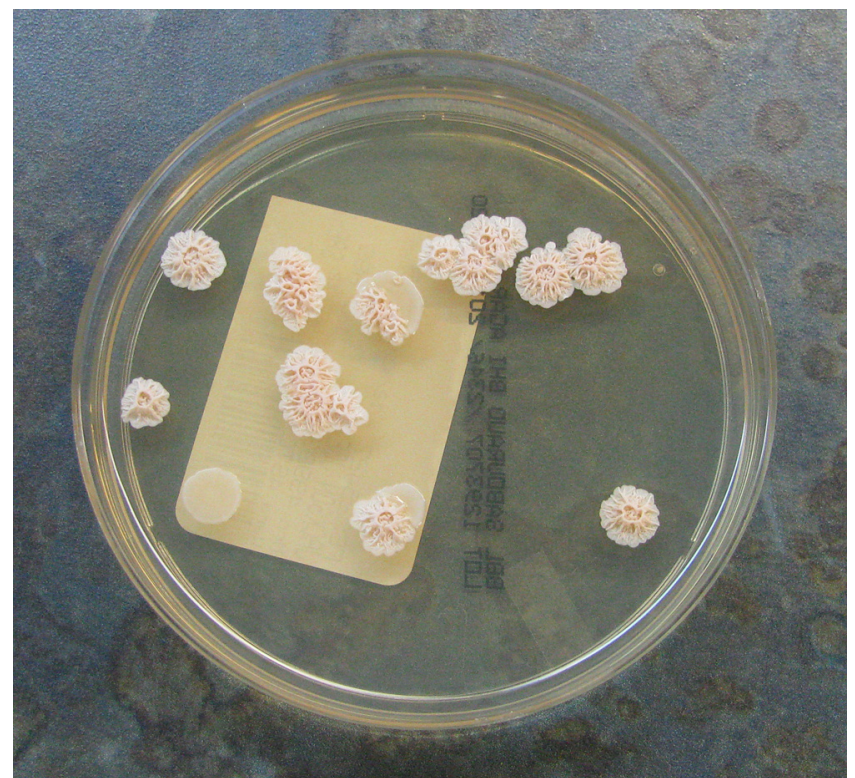

FIG 1 Colony morphology of $N$. neocaledoniensis. Note pinkish hue to the colonies. Smooth colonies are coagulase-negative Staphylococcus.

doxycycline with the addition of oral moxifloxacin. The patient remained clinically stable, with improvement in his signs and symptoms.

Operative cultures were obtained from each abscess, and all grew Nocardia with the same phenotypic characteristics (lysozyme $[+]$, urease $[+]$, and negative hydrolysis of casein, tyrosine, and xanthine, a pattern typical of several species within the former Nocardia asteroides complex). In each instance, the organism was recovered on sheep blood agar at $48 \mathrm{~h}$ after incubation at $35^{\circ} \mathrm{C}$. The colonies were chalky white with a pinkish tinge (Fig. 1), and the organisms were modified acid-fast stain positive. Each culture also exhibited the same susceptibility pattern (Table 1) when

Received 29 February 2012 Returned for modification 29 March 2012 Accepted 8 July 2012

Published ahead of print 11 July 2012

Address correspondence to Robert Fader, rfader@swmail.sw.org

Copyright @ 2012, American Society for Microbiology. All Rights Reserved. doi:10.1128/JCM.00559-12 
TABLE 1 Susceptibility results of the clinical isolate of $N$. neocaledoniensis by broth microdilution

\begin{tabular}{lll}
\hline Antibiotic $(\mathrm{s})$ & $\mathrm{MIC}(\mathrm{s})(\mu \mathrm{g} / \mathrm{ml})$ & Interpretation \\
\hline Amikacin & $\leq 1$ & $\mathrm{~S}$ \\
Amoxicillin/clavulanate & $\geq 64 / 32$ & $\mathrm{R}$ \\
Cefepime & 8 & $\mathrm{~S}$ \\
Ceftriaxone & $\leq 4$ & $\mathrm{~S}$ \\
Ciprofloxacin & 2 & $\mathrm{I}$ \\
Clarithromycin & 2 & $\mathrm{~S}$ \\
Doxycycline & 1 & $\mathrm{~S}$ \\
Imipenem & $\leq 2$ & $\mathrm{~S}$ \\
Linezolid & $\leq 1$ & $\mathrm{~S}$ \\
Moxifloxacin & 0.5 & $\mathrm{~S}$ \\
Tobramycin & $\leq 1$ & $\mathrm{~S}$ \\
Trimethoprim-sulfamethoxazole & $1 / 9$ & $\mathrm{~S}$ \\
\hline
\end{tabular}

tested with a Sensititre rapid growing mycobacterial susceptibility panel (TREK Diagnostic Systems, Cleveland, OH) by using Clinical and Laboratory Standards Institute (CLSI) guidelines (3) and incubated at $30^{\circ} \mathrm{C}$ for 3 days. Pathology was negative for malignancy and fungal organisms.

Sequence-based identification was performed by sequencing of the complete 16S rRNA gene and amino acid sequencing of the secAl gene $(5,6)$ according to standard methods $(4)$. A sequence comparison using the RipSeq Single (Isentio US LLC, Sunnyvale, CA) G1 bacterial database, which contains all published references from valid species, indicated that the isolate was a member of an unusual species of Nocardia ( $N$. neocaledoniensis). The isolate shared 1,390 and 1,397 bp of identity with no gaps (99.5\% identity) with sequences of the DSM 4417T Nocardia neocaledoniensis type strain (GenBank accession no. JF797311 and GQ85380) (2) by complete $16 \mathrm{~S}$ rRNA gene sequence analysis. An identity of $\geq 99.5 \%$ with the $16 \mathrm{~S}$ rRNA gene is considered adequate for a species identification for Nocardia (4). By secA1 analysis, the isolate showed 99\% amino acid identity (one amino acid mismatch), which meets the species definition of the gene analysis (4). The nearest other validated species type strain by complete $16 \mathrm{~S}$ rRNA gene sequencing and secA1 analysis was $N$. thailandica. The $16 \mathrm{~S}$ rRNA and secAl gene sequences have been submitted to GenBank.

Nocardia species are Gram-positive, variably acid-fast, strictly aerobic bacteria that form branched filaments (7). Transmission is mainly from inhalation or direct contact, and the bacteria have been reported to cause cutaneous, subcutaneous, lymphocutaneous, central nervous system, pulmonary, and systemic infections (1). Immunocompromise has been noted in numerous reports as a risk factor for nocardiosis. These characteristics correlate with the case of our patient, who was a recipient of both steroids and methotrexate for at least 10 years to treat rheumatoid arthritis.

In 2004, Saintpierre-Bonaccio et al. (9) reported the phenotypic and genotypic characteristics that distinguished $N$. neocaledoniensis from other strains of Nocardia. This organism was iso- lated from soil in New Caledonia. N. neocaledoniensis has been isolated from clinical specimens obtained from three patients with conjunctivitis, although its role as a pathogen was questioned (10). The organism has also been implicated in mastitis outbreaks among Italian dairy herds (8). Our patient was found to have $N$. neocaledoniensis skin and soft tissue infection, identified through phenotypic and genotypic characteristics, which he may have contracted through occupational or recreational exposure. The initial unsatisfactory response of this patient to oral trimethoprim-sulfamethoxazole as an outpatient may have been secondary to the abscess formation requiring surgical drainage. The susceptibility of this isolate is documented in Table 1. He improved upon treatment with oral trimethoprim-sulfamethoxazole plus intravenous imipenem in conjunction with surgical debridement and later with oral doxycycline and moxifloxacin. To the best of our knowledge, this is the first report of human skin and soft tissue infection caused by N. neocaledoniensis and provides evidence of this species as a human pathogen. It is likely that newer pathogenic species of Nocardia will continue to emerge with the use of sequencing for organism identification.

Nucleotide sequence accession number. Sequence data for the isolate reported in this article have been submitted to GenBank under accession numbers JX297205 (secA1) and JX297206 (16S rRNA).

\section{ACKNOWLEDGMENT}

We thank Steven McNulty and Susan Howard in the Mycobacteria/Nocardia Laboratory at the University of Texas Health Science Center at Tyler for his assistance in submitting the patient isolate to GenBank.

\section{REFERENCES}

1. Brown-Elliott BA, Brown JM, Conville PS, Wallace RJ, Jr. 2006. Clinical and laboratory features of the Nocardia spp. based on current molecular taxonomy. Clin. Microbiol. Rev. 19:259-282.

2. Cloud JL, et al. 2004. Evaluation of partial 16 S ribosomal DNA sequencing for identification of Nocardia species by using the MicroSeq 500 system with an expanded database. J. Clin. Microbiol. 42:578-584.

3. CLSI. 2011. Susceptibility testing of mycobacteria, nocardia, and other aerobic actinomycetes; approved standard-2nd ed. CLSI document M24 A-2 (ISBN 1-56238-746-4). Clinical and Laboratory Standards Institute, Wayne, PA.

4. CLSI. 2008. Interpretive criteria for microorganism identification by DNA target sequencing. Approved guideline. CLSI document MM18-A. Clinical and Laboratory Standards Institute, Wayne, PA.

5. Conville PS, Zelazny AM, Witebsky FG. 2006. Analysis of secA1 gene sequences for identification of Nocardia species. J. Clin. Microbiol. 44: $2760-2766$.

6. Kong F, et al. 2010. secA1 gene sequence polymorphisms for species identification of Nocardia species and recognition of intraspecies genetic diversity. J. Clin. Microbiol. 48:3928-3934.

7. Lerner PI. 1996. Nocardiosis. Clin. Infect. Dis. 22:891-905.

8. Pisoni G, et al. 2008. Short communication: outbreak of Nocardia neocaledoniensis mastitis in an Italian dairy herd. J. Dairy Sci. 91:136-139. doi:10.3168/jds.2007-0477.

9. Saintpierre-Bonaccio D, Maldonado LA, Amir H, Pineau R, Goodfellow M. 2004. Nocardia neocaledoniensis sp. nov., a novel actinomycete isolated from a New-Caledonian brown hypermagnesian ultramafic soil. Int. J. Syst. Evol. Microbiol. 54:599-603.

10. Yin X, et al. 2007. Ocular nocardiosis: HSP65 gene sequencing for species identification of Nocardia spp. Am. J. Ophthalmol. 144:570-573. 